\title{
What patients want and need to know about atrial fibrillation
}

This article was published in the following Dove Press journal:

Journal of Multidisciplinary Healthcare

2 November $201 \mathrm{I}$

Number of times this article has been viewed

\section{Pamela J McCabe}

Saint Mary's Hospital, Mayo Clinic, Rochester, MN, USA
Correspondence: Pamela J McCabe Department of Nursing, Mayo Clinic, Saint Mary's Hospital Domitilla 4D 4-290, Mayo Clinic, 200 First Street SW, Rochester, MN 55905, USA

Tel +l 5072552797

Fax + I 5072554219

Email mccabe.pamela2@mayo.edu
Abstract: Clinicians in a variety of settings are called upon to care for patients diagnosed with atrial fibrillation (AF), a common chronic condition that affects up to 3 million people in the USA. Evidence-based guidelines provide clinicians with direction for treatment of AF, but recommended content for educating patients and counseling about self-management of AF is not included in published guidelines. When patients believe they have a good understanding of AF they report fewer symptoms, perceive greater control over AF, and attribute less emotional distress to AF. Thus, providing patients with information about AF and how to manage it is important for promoting positive outcomes. The purpose of this article is to offer evidence-based recommendations for content to include in self-management education and counseling for patients with AF. Approaches for educating and counseling patients related to AF pathophysiology, the nature of AF (its cause, consequences, and trajectory), treatments, action plans, and symptom management, and managing the psychosocial challenges of living with AF, are discussed.

Keywords: atrial fibrillation, patient education, self-management education, counseling

\section{Introduction}

Atrial fibrillation (AF) is a chronic illness that affects at least 3 million people in the USA and is expected to affect nearly 8 million by $2050 .{ }^{1}$ Thus, a substantial number of health care providers will encounter patients requiring treatment for their $\mathrm{AF}$ and counseling for self-management of AF. Patients who report that they have a good understanding of their AF are more likely to: report fewer symptoms related to AF, perceive greater control over their AF, report greater acceptance of their AF, use more effective coping strategies, and endorse less negative emotions related to their $\mathrm{AF}^{2}$ Thus, assisting patients to understand the nature of AF and how to live with it is critical to promote positive outcomes. Although a large body of information is available to guide clinicians in the options for treatment of $\mathrm{AF}^{3}$ there are few evidence-based resources available to guide clinicians about recommended content for educating patients about $\mathrm{AF}$ and counseling them as they face the challenges of living with AF. The purpose of this article is to offer recommendations for content to include in self-management education and counseling for patients with AF.

In comparison with coronary artery disease (CAD), myocardial infarction, and stroke, AF is less well-known to the public. Research participants reported that they had never heard of AF before being diagnosed with it and were unaware of its symptoms and consequences. ${ }^{4}$ Patients recently diagnosed with AF have knowledge deficits 
about what $\mathrm{AF}$ is, its disease trajectory, cause, consequences, treatment options, the management of risks for progression of AF, when to seek additional evaluation and treatment for symptoms and recurrent episodes of $\mathrm{AF}^{5,6}$ and how to manage its potential challenges to quality of life. ${ }^{4}$

In contrast to the large body of research that has been generated to guide the practice of educating patients with $\mathrm{CAD}^{7}$ or heart failure ${ }^{8}$ to manage their illness, there is limited evidence to guide clinicians about content of an educational program that will prepare patients to effectively self-manage AF. The author's 25 years of clinical experience caring for patients with AF, content published by the Heart Rhythm Society ${ }^{9}$ and American Heart Association, ${ }^{10,11}$ and findings of a few descriptive research studies ${ }^{2,4-6,12,13}$ form the basis of the recommendations offered in this article. Recommendations for education and counseling content include categories related to: (1) pathophysiology; (2) the nature of AF: its cause, consequences and trajectory; (3) treatments; (4) action plans; (5) symptom management; and (6) managing the psychosocial challenges of living with AF.

What patients want and need to know about their illness varies individually and by the context of the health care encounter. Patients' beliefs about their illness influence their coping responses to their illness, which, in turn, influence the outcomes of care, such as adherence to the treatment regimen, ability to function, and psychological adjustment to the illness. ${ }^{14-16}$ Thus, information provided needs to be based not only on an assessment of the patient's readiness to learn, cognitive abilities, health literacy level, and preferred learning style but also on the patient's existing beliefs about the cause, consequences, controllability, duration of symptoms, and diagnosis of AF. Furthermore, because information provided during previous clinical encounters may not have been retained, ${ }^{5}$ reinforcement of that information during subsequent encounters is often necessary. It is important that providers communicate with one another by thoroughly documenting the content of educational/counseling discussions and the patient's response to the discussions. Doing so allows subsequent providers to know what has been discussed so that they can reinforce or add to information previously covered.

\section{Education and counseling components Disease process information is only the beginning}

Research conducted with participants diagnosed with CAD suggests that patients want to know the physiological and pathological basis for their symptoms and condition, ${ }^{17}$ conveyed in plain language. Patients reported that knowing what AF is and how it relates to their symptoms is helpful as a background but emphasized that meaningful patient education must also address questions such as "What caused this?" (cause) "How will this affect my life?" (consequences), and "What can be done about it?"(controllability).,12

\section{The nature of atrial fibrillation: cause information}

Patients' beliefs about the cause of an illness influence both how they manage the illness and their psychological response to the illness. Being able to identify a cause of the illness provides patients with a direction for action plans to control the illness. ${ }^{16}$ Patients who have AF want providers to discuss factors that cause AF and promote its progression, and offer recommendations for modifying factors that might trigger AF and foster persistence of their AF. In some cases, such as old age, the cause is nonmodifiable, or, in AF without comorbidities, the cause is unknown. Inability to identify a modifiable cause for the AF can be emotionally distressing to patients, and some will make efforts to exert control by seeking to find a cause or trigger for recurrent AF episodes. Despite making adjustments to diet and activities, the patient may still be unsuccessful in avoiding recurrent episodes of AF. ${ }^{12}$ If the patient has been adherent to treatment recommendations, it is important for providers to reassure patients that it is not something they have or have not done to cause the AF to recur. For some patients, the inability to control recurrent episodes of $\mathrm{AF}$ is seen as a personal failure. ${ }^{12}$ These patients may need more than the usual reassurance and counseling to help them restructure their beliefs about the nature of AF to gain an acceptance that will support adjustment to their illness.

The link between perceived stress as a trigger for and maintenance of AF has not been well studied, but stress as a cause for $\mathrm{AF}$ is frequently reported by patients in the clinical setting and research participants. ${ }^{2,4,6}$ Patients who held the belief that AF was caused by stress and worry were more likely to endorse negative emotions such as anxiety and depression. ${ }^{2}$ If an inquiry about the patient's beliefs regarding the causes of the original AF and triggers reveal stressrelated beliefs, a referral for stress management education or cognitive behavioral therapy (CBT) may be helpful for those patients. Research participants reported that learning skills of acceptance, reprioritization, learning to say no to demands of others, and "expectation management" helped 
them to reduce stress and adjust more successfully to living with $\mathrm{AF}$ as a chronic and recurrent condition. ${ }^{12}$

If patients who have modifiable conditions that may promote progression of $\mathrm{AF}$ believe their AF is simply due to age, heredity, or chance, it is important for the provider to modify those beliefs by educating them about lifestyle behaviors or comorbid conditions that require modification and management. A full discussion of the management of risk factors for $\mathrm{AF}$ is beyond the scope of this paper, but patients need to know how unmanaged conditions such as hypertension, diabetes, hyperlipidemia, ${ }^{18}$ obstructive sleep apnea, and obesity ${ }^{19}$ contribute to the development of AF and its progression. Providers should emphasize the role that untreated risk factors play in undermining current AF treatments and the patient's opportunity for exerting some control over the AF through management of those conditions.

\section{The nature of atrial fibrillation: consequences and trajectory of AF}

Patients' beliefs about the consequences and trajectory of their illness influence their functional and psychological response to their illness. ${ }^{14}$ For example, research participants who presented at the emergency department with abrupt symptoms of chest pain and palpitations believed that cause of their symptoms was a heart attack or life threatening condition because the symptoms were related to the heart. Their perceptions about the consequences of having a heart attack led to fears of death and disability. Their anxiety was substantially reduced when the physician or nurse informed them they were not having a heart attack. ${ }^{4}$ Correction of misperceptions related to the cause of the symptoms and perceived consequences, as well as providing reassurance about the ability to control the symptoms, is an important educational intervention in the early phase of the AF trajectory.

In contrast, some patients experienced less abrupt symptoms, such as fatigue or dyspnea, for months. Their AF was detected when they were seen by a provider for a routine examination or another complaint. In this case, study participants reported that it was important for the provider to convey the serious nature of $\mathrm{AF}$ and its consequences and the importance of seeking treatment. Some patients failed to pursue treatment when AF episodes were recurrent because they perceived little concern from their provider and therefore concluded that if the provider was not concerned, they should not be concerned. Other patients reported that the lack of interest expressed by their provider for their symptoms and recurrent AF was distressing. Patients described the provider as having a dismissive attitude that made them feel their concerns about AF were inconsequential and unimportant. The above provider responses were associated with delays in receiving advanced therapy for recurrent AF, strained the provider-patient relationship, and sometimes resulted in the patient seeking a new provider. ${ }^{4,12}$

Research participants reported that it was important for providers to convey balanced information about the serious nature of AF. Many found it helpful to be told that AF "was not going to kill them." This relieved anxiety and helped them to tolerate and manage symptoms more effectively at home. ${ }^{4}$ Although patients want clinicians to offer encouragement that AF can be managed, they also emphasize that providers need to convey the serious consequences of untreated AF: risk of stroke, heart failure, or progression of AF.

Providing the patient with a realistic expectation about the trajectory of $\mathrm{AF}$ - that, for most patients, $\mathrm{AF}$ is a recurrent and chronic illness - is an important factor in supporting patients' adjustment to AF. Patients whose first AF episode is managed by cardioversion alone may develop the perception that their AF was an acute isolated event that was "taken care of" by the cardioversion. Koponen et $\mathrm{al}^{6}$ reported that $27 \%$ of patients who were discharged from the emergency room after treatment for AF believed that recurrence of treated $\mathrm{AF}$ was rare. If patients leave the care encounter with the belief that $\mathrm{AF}$ will not recur, they may be distressed when they experience a recurrent episode of AF. ${ }^{12}$ Patients report that it is important for providers to inform them the AF may recur again suddenly and that they will need to return for additional treatment. This anticipatory guidance gives the patient the knowledge they need to cope more effectively if and when another episode of AF occurs.

\section{Control and treatment information}

Study participants reported that a full discussion of treatment options, with rationale for each, was important. When they understood that AF may recur, they wanted to know that there was "something else that can be done." Some patients were distressed by hearing different opinions among providers who did not provide rationales for their recommendations. ${ }^{4,12}$ Patients who discuss AF with one another may not realize the importance of individualized treatment provided according to type of AF and with consideration of each patient's clinical condition. Thus, it is important to provide information about why a particular treatment is being recommended 
for the patient at this particular phase in their trajectory of AF and why another treatment option is not recommended. Providing information about the treatment, its benefits, risks, and side effects in a manner that is encouraging, yet clear about its limitations, will help the patient to develop realistic expectations that support positive adjustment. Without such information, patients come to their own conclusions, may not understand the difference between a rate-control versus a rhythm-control strategy, ${ }^{5}$ and may have unrealistic expectations for the outcome of the plan of care. Koponen et $\mathrm{al}^{6}$ reported that 3 months after treatment in the emergency room $67 \%$ of participants believed that AF would not recur if they were taking medication to inhibit it. Patients who do not understand the limitations of drug or ablation therapy may become very distressed if they have AF episodes even though they are being treated with medications and/or ablation.

Patients who are at moderate to high risk for stroke will likely require warfarin or other anticoagulant therapy for stroke prevention. Education and counseling to support patients with moderate to high risk for stroke to gain the knowledge and skills for self-management for safe anticoagulant therapy will need to be added to the discussion regarding strategies for AF rate or rhythm control. A discussion of the recommended content for patient education regarding warfarin use is beyond the scope of this paper but is available elsewhere..$^{20,21}$

\section{Action plans}

Patients want direction regarding actions to take in response to symptoms and possible adverse responses or lack of response to treatment. A mutual understanding between the patient and provider regarding the treatment goal must be achieved before developing the action plan.

Because symptoms alone are not adequate for determining effectiveness of therapy, ${ }^{22}$ pulse taking is an important strategy for monitoring response to treatment whether the goal is rate control or maintenance of sinus rhythm. Patients should be taught how to palpate their pulse, count its rate, and monitor the pattern for irregularity. ${ }^{13}$ Palpating the pulse is favored over electronic readings because electronic methods do not provide information about the regularity of the pulse and may provide inaccurate rates if the patient is in AF. If the patient is unable to palpate the pulse, patients who are willing to learn to auscultate their heart for rhythm and rate can be taught to do so with a stethoscope that can be purchased for US\$10-15 at home medical supply or drug stores. If the treatment goal is to maintain sinus rhythm, the patient should be counseled about the actions to take if the pulse becomes irregular, which may indicate return of AF. If rate control is the goal, patients should be informed about the target range for their pulse rate and what actions should be taken if symptoms are not controlled or heart rate is outside of the target goal range.

Patients need guidance regarding the type of provider/ setting they should contact for evaluation/treatment assistance for specific symptoms; for example, which symptoms require a call to 911 for emergency services versus those that could be monitored and managed with a visit or telephone call to their provider. When AF is diagnosed, patients should be educated about signs and symptoms of stroke and the importance of seeking emergency services promptly if stroke signs or symptoms occur. Because this critical information may not be retained after initial communication, ${ }^{5,6}$ reinforcement of this information should occur on subsequent clinical encounters.

\section{Symptom management}

Despite the best attempts to treat AF with medications and or ablation procedures, some patients continue to experience intermittent or persistent symptoms they attribute to AF. Furthermore, some patients attribute symptoms to AF even when objective reports show AF is not present. ${ }^{22}$ Thus, education and counseling regarding symptom-management strategies is an important intervention to promote quality of life for patients with AF. Decreased energy level and endurance and shortness of breath, known to clinicians as fatigue and dyspnea, respectively, are symptoms frequently attributed to AF and reported as having a high symptom burden among patients with recurrent symptomatic AF. ${ }^{4}$ Management of fatigue and dyspnea in the context of AF is critically understudied and evidence-based interventions directed specifically toward patients with AF are not established. However, reports of patients who participated in a qualitative descriptive study ${ }^{12}$ and recommendations offered for patients with other chronic illnesses who experience fatigue ${ }^{23}$ or dyspnea ${ }^{24}$ provide some guidance that may be useful until knowledge specific to patients with AF can be generated.

Patients who experience persistent fatigue in the context of optimal treatment should be evaluated for conditions other than AF that may be contributing to fatigue. Obstructive sleep apnea is one of the common contributors to fatigue in patients with AF. Screening for obstructive sleep apnea can be accomplished using the Berlin Questionnaire ${ }^{25}$ and, if positive, patients should be referred for polysomnography. ${ }^{26}$ If an assessment reveals that fatigue is simply related to poor sleep quality resulting from the patient's behaviors and habits, 
sleep hygiene counseling may provide the patient with selfmanagement strategies to improve sleep. ${ }^{23}$

Because depression may be a contributor to fatigue, patients should be assessed for depression and treated. ${ }^{23}$ Patients who reported more symptoms of anxiety and emotional distress related to $\mathrm{AF}$ also reported more symptoms of fatigue and less vigor. ${ }^{4}$ Although the benefit of CBT for managing fatigue in patients with $\mathrm{AF}$ has not been studied, research in other patient populations where CBT was used to treat fatigue suggest that CBT could be beneficial for fatigue management in patients with AF. Other fatigue management strategies reported by patients included: acceptance of a different level of activity tolerance; prioritizing activities - giving up activities that they did not value; restructuring expectations about the amount of time devoted to accomplishing the project/task; taking more rest breaks between activities requiring physical and mental exertion; delegating work to others; and increasing hours of sleep. ${ }^{12}$

Providers should discuss with their AF patients the benefit of regular exercise for managing fatigue. Although evidence is limited, small studies reported that AF patients participating in aerobic exercise and muscle strengthening reported greater vitality and physical functioning, improved emotional health, and less symptom severity. ${ }^{27-30}$ Some patients with AF become reluctant to engage in exercise for fear of triggering an $\mathrm{AF}$ episode or because they perceive themselves as too exhausted to exercise. ${ }^{12}$ Patients should be informed that exercise is safe, beneficial, and may actually promote an electrophysiologic environment that reduces the potential for triggered electrical activity which can induce $\mathrm{AF}^{27}$ Patients who are very deconditioned or afraid of beginning an exercise program may benefit by a referral to a cardiac rehabilitation program for exercise training instruction and a short-term program of supervised exercise. Referral to a cardiac rehabilitation program should not be withheld simply because of third-party payer concerns. Some patients who have private insurance may have coverage for payment or some patients may be willing to pay out of pocket costs for a Phase III program that provides supervised exercise at lower costs than the Phase II programs provided for patients who require more monitoring and supervision during exercise.

Patients with symptomatic recurrent AF who reported more symptoms of fatigue also reported more dyspnea; ${ }^{4}$ thus, interventions for the management of fatigue already discussed may be useful for reducing dyspnea as well. ${ }^{24}$ Patients with symptomatic $\mathrm{AF}$ who reported greater symptom burden related to shortness of breath reported more symptoms of anxiety. ${ }^{4}$ Because anxiety can enhance the sensation of dyspnea, use of self-management anxiety reduction strategies such as distraction and relaxation may promote an increased sense of control over the dyspnea. ${ }^{24}$ Patients reported that learning to live with activity limitations and energy conservation strategies such as pacing activities and stopping to rest frequently were helpful for managing their shortness of breath. ${ }^{12}$ Patients who have a chronic pulmonary illness in addition to their AF may benefit from participation in a pulmonary rehabilitation program.

\section{Managing psychosocial challenges of living with AF}

Some patients experience symptoms of depression and anxiety that they associate with the challenges of living with the symptoms and unpredictable nature of recurrent AF. Research participants expressed discouragement and demoralization when function-limiting symptoms and uncertainty associated with $\mathrm{AF}$ recurrence caused them to curtail valued activities and give up important goals. ${ }^{12}$ The true prevalence of depression and anxiety in patients with AF is unknown, but in study participants recruited from referral settings, up to $38 \%$ of participants' scores on instruments to measure psychological distress were in the clinically significant range ${ }^{31}$ or mean scores were greater than those of the control sample means. ${ }^{32}$ Thus, providers should assess AF patients for psychological distress and provide treatment and referrals based on those assessments. Most patients' emotional needs can be addressed by providers who clearly explain the situation and options, actively listen to concerns, acknowledge the challenges of living with $\mathrm{AF}$ and treatment limitations, and show a caring attitude and willingness to refer if needed. Patients who have clinical depression or anxiety disorder should be treated and/ or referred to a psychiatrist if the provider believes specialty care is needed. Patients who are having difficulty coping may benefit from a referral to a therapist for CBT.

The effect of living with AF on the patient's family/social dynamics has not been well studied, but patients do report that AF has consequences for those close to them. ${ }^{2}$ Some research participants reported that the functional limitations and emotions they experienced strained family and social relationships. Perceived lack of support and understanding from family members and coworkers in regard to how symptoms affected their ability to carry out usual home, social, and work activities were described. Even after being diagnosed with AF, participants worried that others would think they were shirking responsibilities and feared being labeled as "lazy" or "hypochondriacs" because family and coworkers did not perceive AF as a serious health problem. ${ }^{12}$ If the patient allows family members to be present during 
clinical visits, providers should include family in discussions about AF, its symptoms and consequences, and realistic treatment goals. This information may help family members provide effective support for patients. As noted above, CBT may help patients learn strategies to promote healthy social relationships and to set realistic goals for meeting the demands of their family, social, and work roles.

Some patients seek Internet resources for education and support, especially if they perceive information provided face-to-face by their provider is incomplete or not in line with their goals. If patients and family members wish to access Internet resources, they should be counseled to seek information from accurate and current sources that are supported and maintained by credible institutions or organizations. There are an abundance of websites dedicated to AF, but not all contain accurate and helpful information. The clinician may want to inquire if the patient has used Internet resources and, if so, what perceptions patients have developed from information they retrieved. If the Internet visits have led to inaccurate perceptions, the clinician can address the inaccuracies. This author does not endorse specific websites, but patient information about AF can be found on the American Heart Association, Heart Rhythm Society, and many major medical center websites. A website known as StopAfib.org, ${ }^{33}$ created specifically for patients and families, is maintained by an individual but is well-known by experts in electrophysiology, some of whom are listed on the board of directors of the site.

\section{Conclusion}

$\mathrm{AF}$ is a common chronic illness that requires patients to actively partner with providers to achieve optimal treatment outcomes. Patients who have a good understanding of their AF report greater acceptance of their AF, fewer symptoms, and less negative emotions related to their AF. Providers should engage patients as partners in their care by educating and counseling them to gain an understanding of the nature of AF, its treatments, and self-care activities that will lead to positive outcomes. Knowledge about the patients' perceptions regarding the cause, consequences, and controllability of their AF help to guide education and counseling discussions. This article has discussed key educational and counseling interventions that have been based on limited data from descriptive research and included in resources published by the American Heart Association and Heart Rhythm Society. Further research is needed to determine the best approaches and content for AF patient education. Current data and resources support including educational content related to pathophysiology, the nature of AF (its cause, consequences, and trajectory) treatments, action plans, and symptom management, and managing the psychosocial challenges of living with AF.

\section{Take-home points for AF patient education}

Keep pathophysiology information basic and use plain language. Find out how much the patient wants to know and tailor information accordingly. Make connections between the physiological mechanisms of AF and the patient's symptoms. Provide information by verbal explanation and written and video resources according to the patient's preferences.

\section{Causes of AF}

Emphasize that the cause may be difficult to determine. If modifiable risk factors are present, reinforce the importance of making lifestyle changes to manage the risk factors that can undermine the benefit of AF treatment.

\section{Consequences}

Provide balanced information. Emphasize that AF has serious consequences and requires individualized treatment. Acknowledge that symptoms can be frightening, but they generally do not signify a life-threatening condition.

\section{Course of AF}

Provide explanation of the types of AF and natural course of $\mathrm{AF}$ - that generally it is progressive and chronic. Recurrences are common, despite the patient's best efforts to adhere to treatment.

\section{Treatment}

Treatment is essential, but treatments vary by individual and type of AF. Treatments often produce an incomplete result or fail altogether. Discuss realistic treatment goals.

\section{Action planning}

Ensure that the patient understands the treatment goal and actions to take if the treatment goal is not met or if adverse effects of treatment occur. Assessment of heart rate and rhythm is an important aspect of self-monitoring the response to treatment.

\section{Psychosocial responses to $\mathrm{AF}$}

Assess patients' perceptions about the effects of AF on their psychosocial well-being. Acknowledge concerns and encourage realistic expectations regarding goals of therapy. Patients who have a persistently negative appraisal regarding the effect of AF on their life and difficulty coping with challenges may benefit from psychiatric assessment and cognitive behavioral interventions. 


\section{Disclosure}

The author reports no conflicts of interest in this work.

\section{References}

1. Nacarelli GV, Varker H, Lin J, Schulman KL. Increasing prevalence of atrial fibrillation and flutter in the United States. Am J Cardiol. 2009; 104(11):1534-1539.

2. McCabe PJ, Barnason SA, Houfek J. Illness beliefs in patients with recurrent symptomatic atrial fibrillation. Pacing Clin Electrophysiol. 2011;34(7):810-820.

3. Fuster V, Ryden LE, Cannom DS, et al. ACC/AHA/ESC 2006 Guidelines for the Management of Patients with Atrial Fibrillation: a report of the American College of Cardiology/American Heart Association Task Force on Practice Guidelines and the European Society of Cardiology Committee for Practice Guidelines (Writing Committee to Revise the 2001 Guidelines for the Management of Patients With Atrial Fibrillation): developed in collaboration with the European Heart Rhythm Association and the Heart Rhythm Society. Circulation. 2006;114(7):e257-e354.

4. McCabe PJ. Predictors of Symptoms and Psychological Distress in Patients with Recurrent Symptomatic Atrial Fibrillation [doctoral dissertation]. Omaha, NE: University of Nebraska Medical Center; 2009.

5. McCabe PJ, Schad S, Hampton A, Holland DE. Knowledge and self-management behaviors of patients with recently detected atrial fibrillation. Heart Lung. 2008;37(2):79-90.

6. Koponen L, Rekola L, Ruotsalainen T, Lehto M, Leino-Kilpi H, VoipioPulkki LM. Patient knowledge of atrial fibrillation: 3 month follow-up after an emergency room visit. J Adv Nurs. 2007;61(1):51-61.

7. Dusseldorp E. van Elderen T, Maes S, Meulman J, Kraaij VA. A metaanalysis of psychoeducational programs for coronary heart disease patients. Health Psychol. 1999;18(5):506-519.

8. Fredericks S, Beanlands H, Spalding K, DaSilva M. Effects of the characteristics of teaching on the outcomes of heart failure patient education: A systematic review. Eur J Cardiovasc Nurs. 2010;9(1):30-37.

9. hrsonline.org. Washington, DC: Heart Rhythm Society. Patient information Atrial Fibrillation. Available at: http://www.hrsonline.org/ PatientInfo/HeartRhythmDisorders/AFib/index.cfm. Accessed July 12, 2011.

10. American Heart Association. Atrial fibrillation [web page on the Internet]. Dallas, TX: American Heart Association; 2011 [updated 2011 June 10]. Available at: http://www.heart.org/HEARTORG/Conditions/ Arrhythmia/AboutArrhythmia/Atrial-Fibrillation-AF_UCM_302027_ Article.jsp. Accessed July 5, 2011.

11. Shea JB, Sears SF. A patient's guide to living with atrial fibrillation. Cardiology Patient Page. Circulation. 2008;117(20):e340-e343.

12. McCabe PJ, Schumacher K, Barnason SA. Living with atrial fibrillation: A qualitative study. J Cardiovasc Nurs. 2011;26(4):336-344.

13. Munschauer FE, Sohocki D, Smith Carrow S, Priore RL. A community education program on atrial fibrillation: implications of pulse selfexamination on awareness and behavior. J Stroke Cerebrovasc Dis. 2004;13(5):208-213.

14. Leventhal H, Leventhal E, Cameron LD. Representations, procedures, and affect in illness self-regulation: a perceptive-cognitive model. In: Baum A, Revenson T, Singer J, editors. Handbook of Health Psychology. Mahwah, NJ: Lawrence Erlbaum; 2001:19-47.
15. Hagger MS, Orbell S. A meta-analytic review of the common-sense model of illness representations. Psychol Health. 2003;18(2):141-184.

16. Hirani SP, Newman SP. Patients' beliefs about their cardiovascular disease. Heart. 2005;91(9):1235-1239.

17. Karlik BA, Yarcheski A, Braun J, Wu M. Learning needs of patients with angina: An extension study. J Cardiovasc Nurs. 1990;4(2):70-82.

18. Chamberlain AM, Agarwal SK, Ambrose M, Folsom AR, Solliman EZ, Alonso A. Metabolic syndrome and incidence of atrial fibrillation among blacks and whites in the Atherosclerosis Risk in Communities (ARIC) Study. Am Heart J. 2010;159(5):850-856.

19. Gami A, Hodge DO, Herges RM, et al. Obstructive sleep apnea, obesity and the risk of incident atrial fibrillation. $J$ Am Coll Cardiol. 2007; 49(5):565-571.

20. Zeolla MM, Brodeur MR, Dominelli A, Haines ST, Allie ND. Development and validation of an instrument to determine patient knowledge: the oral anticoagulation knowledge test. Ann Pharmacotherapy. 2006;40(4):633-638.

21. Newall F, Monagle P, Johnston L. Patient understanding of warfarin therapy: A review of education strategies. Hematol. 2005;10(6): 437-442.

22. Mehall JR, Kohut RM, Shcneeberger EW, Merrill WH, Wolf RK. Absence of correlation between symptoms and rhythm in "symptomatic" atrial fibrillation. Ann Thorac Surg. 2007;83(6):2118-2121.

23. Piper BF. Fatigue. In: Carrieri-Kohlman V, Lindsey AM, West CM, editors. Pathophysiological Phenomena in Nursing. 3rd ed. St Louis, MO: Saunders; 2003:209-234.

24. Carrieri-Kohlman V, Stulbarg. Dyspnea. In: Carrieri-Kohlman V, Lindsey AM, West CM, editors. Pathophysiological Phenomena in Nursing. 3rd ed. St Louis, MO: Saunders; 2003:175-208.

25. Netzer NC, Stoohs RA, Netzer CM, Clark K, Strohl KP. Using the Berlin Questionnaire to identify patients at risk for the sleep apnea syndrome. Ann Intern Med. 1999;131(7):485-491.

26. Gami AS, Somers VK. Implications of obstructive sleep apnea for atrial fibrillation and sudden cardiac death. J Cardiovasc Electrophysiol. 2008;19(9):997-1003.

27. Hegbom F, Stavem K, Sire S, Heldal M, Orning OM, Gjesdal K. Short-term exercise training in patients with chronic atrial fibrillation. Effects on exercise capacity, AV conduction and quality of life. J Cardiopulm Rehabil. 2006;26(1):24-29.

28. Hegbom F, Stavem K, Sire S, Heldal M, Orning OM, Gjesdal K. Effects of short-term exercise training in patients with chronic atrial fibrillation. Int J Cardiol. 2007;116(1):86-92.

29. Vanhees L, Schepers D, Defoor J, Bruselle S, Tchursh N, Fagard R. Exercise performance and training in cardiac patients with atrial fibrillation. $J$ Cardiopulm Rehabil. 2000;20(6):346-352.

30. Mertens DJ. Exercise training for patients with chronic atrial fibrillation. J Cardiopulm Rehabil. 2006;26(1):30-31.

31. Thrall G, Lip GYH, Carroll D, Lane D. Depression, anxiety, and quality of life in patients with atrial fibrillation. Chest. 2007;132(4):1259-1264.

32. McCabe PJ, Barnason SA. Negative emotions and symptom burden: What is the relationship in patients with recurrent atrial fibrillation? Heart Rhythm. 2010;7(5 Supp 1):S131.

33. StopAfib.org [homepage on the Internet]. Greenwood, TX: StopAfib.org; 2007-2011 [updated 2010 Nov 22]. Available at: http://www.stopafib. org. Accessed July 12, 2011.
Journal of Multidisciplinary Healthcare

\section{Publish your work in this journal}

The Journal of Multidisciplinary Healthcare is an international, peerreviewed open-access journal that aims to represent and publish research in healthcare areas delivered by practitioners of different disciplines. This includes studies and reviews conducted by multidisciplinary teams as well as research which evaluates the results or conduct of such teams or

\section{Dovepress}

healthcare processes in general. The journal covers a wide range of areas and welcomes submission from practitioners at all levels, from all over the world. The manuscript management system is completely online and includes a very quick and fair peer-review system. Visit http://www.dovepress.com/testimonials.php to read real quotes from published authors. 